\title{
Hydrogenated turpentine: a bio-based component for jet fuel
}

David Donoso $^{1}$, Rosario Ballesteros ${ }^{1}$, David Bolonioº ${ }^{2}$, María-Jesús García-Martínez², Magín Lapuerta $^{1}$ and Laureano Canoira ${ }^{2}$.

${ }^{1}$ ETS Ingeniería Industrial. Universidad de Castilla-La Mancha. Avda. Camilo José Cela s/n, 13071 Ciudad Real, Spain.

${ }^{2}$ Department of Energy \& Fuels, ETS Ingenieros de Minas y Energía, Universidad Politécnica de Madrid. Ríos Rosas 21, 28003 Madrid, Spain.

\section{Supplementary material}

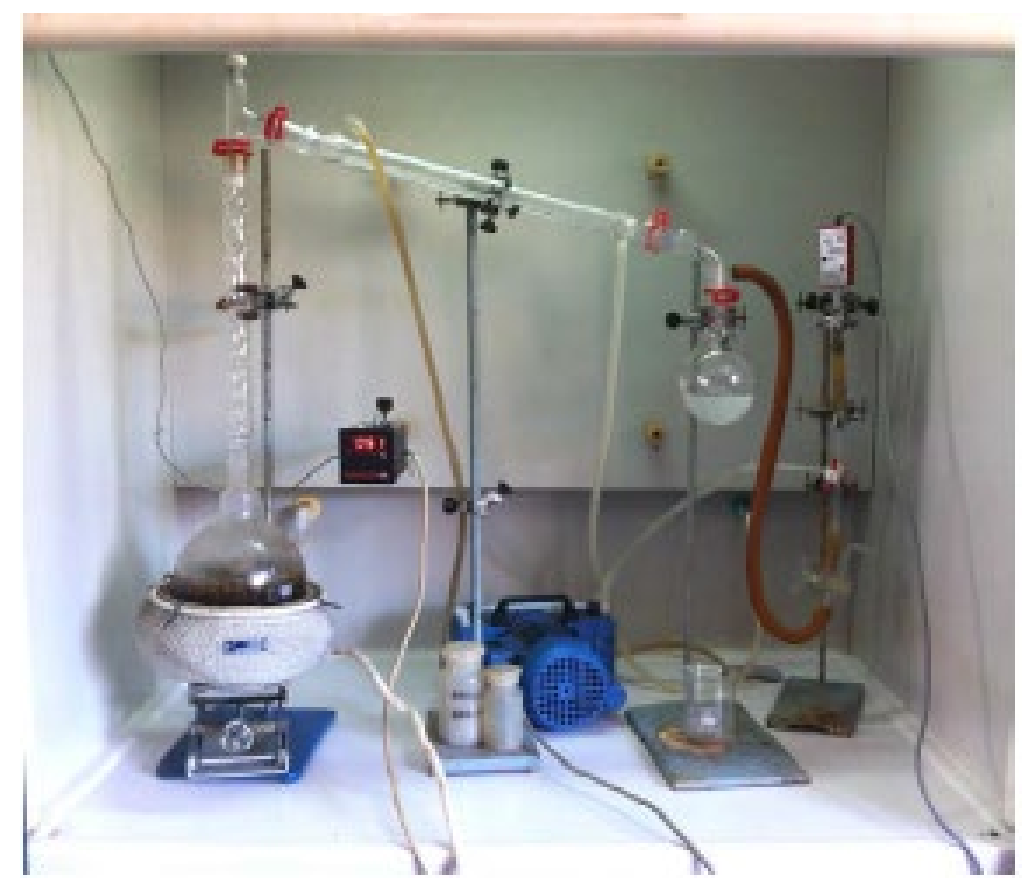

Figure 1S. Vacuum distillation apparatus for resin

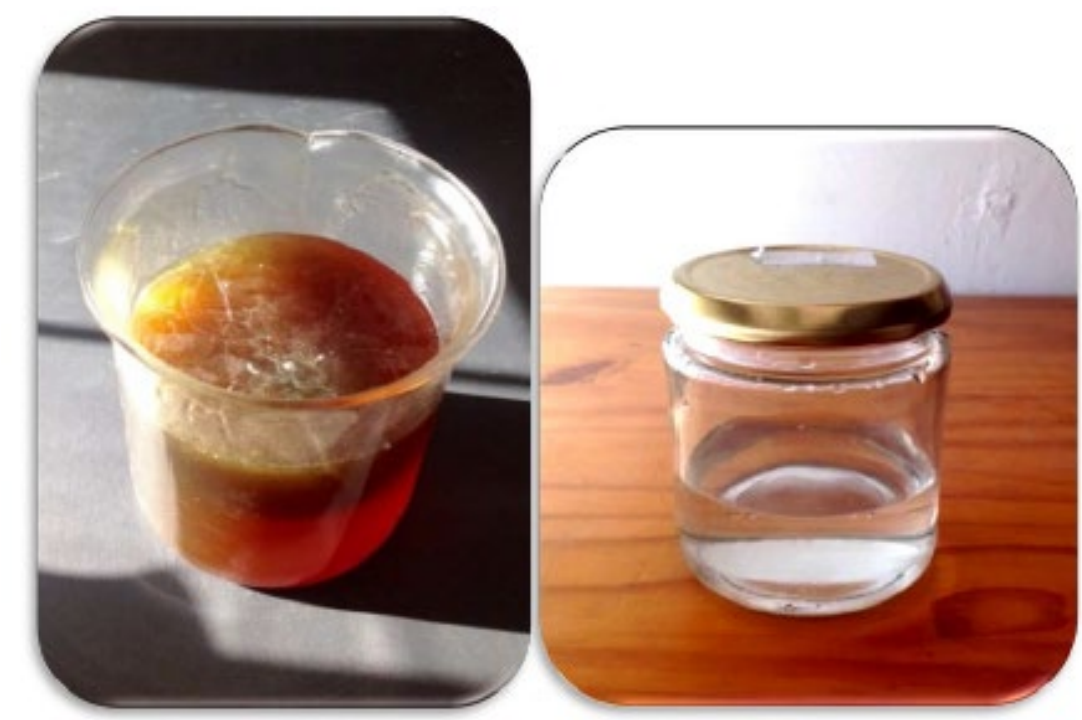

Figure 2S. Vacuum distillation products: rosin (left) and turpentine (right) 
Table 1S. Chemical composition of turpentine obtained from pine resin

\begin{tabular}{|l|l|l|l|}
\hline Compound & Retention time (min) & Quality & Amount (\% w/w) \\
\hline$\alpha$-Pinene & 14.703 & 97 & 67.3 \\
\hline Camphene & 15.330 & 94 & 1.2 \\
\hline$\beta$-Pinene & 16.761 & 96 & 21.5 \\
\hline$\beta$-Myrcene & 17.422 & 83 & 1.0 \\
\hline D-Limonene & 19.021 & 94 & 2.7 \\
\hline --Carene & 21.174 & 97 & 0.4 \\
\hline$\alpha$-Terpineol & 24.879 & 80 & 0.3 \\
\hline$\alpha$-Cubebene & 29.300 & 99 & 0.4 \\
\hline$\alpha$-Longipinene & 29.400 & 99 & 0.2 \\
\hline Copaene & 30.083 & 98 & 0.2 \\
\hline Longifolene & 31.011 & 91 & 2.0 \\
\hline Caryophyllene & 31.260 & 94 & 2.2 \\
\hline$\alpha$-Caryophyllene & 32.169 & 98 & 0.3 \\
\hline Caryophyllene oxide & 35.256 & 91 & 0.3 \\
\hline
\end{tabular}

Table $2 S$. Chemical composition of hydrogenated turpentine at 3\% conversion by GC-MS

\begin{tabular}{|l|l|l|l|}
\hline Compound & Retention time (min) & Quality & Amount (\% w/w) \\
\hline$\alpha$-Pinene & 15.188 & 97 & 67.2 \\
\hline Camphene & 15.845 & 98 & 1.1 \\
\hline$\beta$-Pinene & 17.232 & 96 & 19.9 \\
\hline Pinane (endo, exo) & 17.378 & 96 & 1.8 \\
\hline 2,6-Dimethyl-2,6-octadiene & 17.651 & 87 & 0.5 \\
\hline 1-p-Menthene & 19.219 & 97 & 0.8 \\
\hline D-Limonene & 19.461 & 98 & 1.7 \\
\hline$\alpha$-Terpinolene & 21.599 & 98 & 0.3 \\
\hline$\alpha$-Pinene oxide & 22.071 & 46 & 0.0 \\
\hline Verbenene & 22.475 & 53 & 0.3 \\
\hline L-Pinocarveol & 23.529 & 95 & 0.3 \\
\hline $1(7), 5,8-O-M e n t h a t r i e n e$ & 23.686 & 70 & 0.3 \\
\hline Trans-isolimonene & 25.301 & 90 & 0.5 \\
\hline$\alpha$-Cubebene & 29.722 & 99 & 0.4 \\
\hline$\alpha$-Longipinene & 29.845 & 98 & 0.2 \\
\hline Copaene & 30.509 & 97 & 0.3 \\
\hline Longifolene & 31.446 & 99 & 2.0 \\
\hline Caryophyllene & 31.687 & 99 & 1.9 \\
\hline$\alpha$-Caryophyllene & 32.603 & 98 & 0.3 \\
\hline Caryophyllene oxide & 35.723 & 91 & 0.4 \\
\hline
\end{tabular}


Table 3S. Chemical composition of hydrogenated turpentine at 16\% conversion by GC-MS

\begin{tabular}{|c|c|c|c|}
\hline Compound & Retention time (min) & Quality & Amount (\% w/w) \\
\hline$\alpha$-Pinene & 15.188 & 96 & 64.4 \\
\hline Camphene & 15.845 & 98 & 1.1 \\
\hline$\beta$-Pinene & 17.232 & 96 & 19.9 \\
\hline Pinane (endo, exo) & 17.378 & 97 & 0.9 \\
\hline 2-Ethyl-6-methyl-1,5-heptadiene & 17.514 & 83 & 0.4 \\
\hline 2,6-Dimethyl-2,6-octadiene & 17.651 & 86 & 0.6 \\
\hline 1-p-Menthene & 19.219 & 97 & 1.0 \\
\hline D-Limonene & 19.461 & 98 & 2.0 \\
\hline$\alpha$-Terpinolene & 21.599 & 98 & 0.2 \\
\hline$\alpha$-Pinene oxide & 22.071 & 38 & 0.4 \\
\hline Verbenene & 22.455 & 70 & 0.4 \\
\hline L-Pinocarveol & 23.529 & 90 & 0.3 \\
\hline 1(7),5,8-O-Menthatriene & 23.686 & 62 & 0.4 \\
\hline Trans-isolimonene & 25.301 & 90 & 0.7 \\
\hline Verbenone & 25.664 & 97 & 0.3 \\
\hline$\alpha$-Cubebene & 29.722 & 99 & 0.5 \\
\hline$\alpha$-Longipinene & 29.845 & 98 & 0.3 \\
\hline Copaene & 30.509 & 98 & 0.3 \\
\hline Longifolene & 31.446 & 99 & 2.5 \\
\hline Caryophyllene & 31.687 & 99 & 2.3 \\
\hline$\alpha$-Caryophyllene & 32.603 & 98 & 0.3 \\
\hline Caryophyllene oxide & 35.723 & 90 & 0.7 \\
\hline
\end{tabular}

Table 4S. Chemical composition of hydrogenated turpentine at $26 \%$ conversion by GC-MS

\begin{tabular}{|l|l|l|l|}
\hline Compound & Retention time (min) & Quality & Amount (\% w/w) \\
\hline$\alpha$-Pinene & 15.188 & 96 & 56.8 \\
\hline Pinane (endo, exo) & 16.833 & 97 & 5.7 \\
\hline$\beta$-Pinene & 17.232 & 94 & 2.2 \\
\hline Pinane (endo, exo) & 17.378 & 91 & 21.6 \\
\hline 3-p-Menthene & 17.651 & 94 & 0.3 \\
\hline p-Menthane & 17.873 & 97 & 0.6 \\
\hline 1-p-Menthene & 19.219 & 97 & 2.8 \\
\hline D-Limonene & 19.461 & 96 & 0.3 \\
\hline$\alpha$-Pinene oxide & 22.071 & 43 & 0.5 \\
\hline Verbenene & 22.455 & 38 & 0.4 \\
\hline 1(7),5,8-O-Menthatriene & 23.686 & 49 & 0.7 \\
\hline Trans-isolimonene & 25.301 & 76 & 0.5 \\
\hline Verbenone & 25.664 & 97 & 0.6 \\
\hline$\alpha$-Cubebene & 29.722 & 99 & 0.3 \\
\hline$\alpha$-Longipinene & 29.845 & 99 & 0.3 \\
\hline Copaene & 30.509 & 99 & 0.4 \\
\hline Iridomyrmecin & 31.173 & 46 & 1.0 \\
\hline Longifolene & 31.446 & 99 & 3.6 \\
\hline 7,7-Dimethyl-2-methoxy-2-norbornene & 31.893 & 64 & 0.8 \\
\hline 1-Methyl-1-cycloundecene & 31.983 & 58 & 0.7 \\
\hline
\end{tabular}


Table 5S. Chemical composition of hydrogenated turpentine at 59\% conversion by GC-MS

\begin{tabular}{|l|l|l|l|}
\hline Compound & Retention time (min) & Quality & Amount (\% w/w) \\
\hline$\alpha$-Pinene & 15.064 & 95 & 39.6 \\
\hline 2,6-Dimethyloctane & 15.249 & 78 & 1.0 \\
\hline Pinane (endo, exo) & 16.823 & 97 & 6.6 \\
\hline Isocamphane & 17.220 & 94 & 1.4 \\
\hline Pinane (endo, exo) & 17.418 & 91 & 47.6 \\
\hline p-Menthane & 17.863 & 94 & 0.9 \\
\hline 1-p-Menthene & 19.231 & 97 & 1.1 \\
\hline 3-p-Menthene & 19.437 & 96 & 0.0 \\
\hline Copane & 29.907 & 53 & 0.0 \\
\hline$\alpha$-Longipinene & 29.831 & 98 & 0.0 \\
\hline Copaene & 30.509 & 99 & 0.0 \\
\hline Tetradecyne & 31.227 & 64 & 0.0 \\
\hline (-)-Isolongifolene & 31.446 & 99 & 1.8 \\
\hline
\end{tabular}

Table 6S. Elemental composition of turpentine, its main components, hydrogenated turpentines and Jet A1

\begin{tabular}{|c|c|c|c|}
\hline & $C(\% w / w)$ & $H(\% w / w)$ & $O(\% w / w)$ \\
\hline Turpentine $^{(1)}$ & 88.1 & 11.8 & 0.1 \\
\hline$\alpha$-Pinene & 88.2 & 11.8 & 0.0 \\
\hline$\beta$-Pinene & 88.2 & 11.8 & 0.0 \\
\hline D-Limonene & 88.2 & 11.8 & 0.0 \\
\hline Myrcene & 88.2 & 11.8 & 0.0 \\
\hline HT1 $(3 \%)^{(1)}$ & 88.1 & 11.9 & 0.0 \\
\hline HT2 $(16 \%)^{(1)}$ & 88.0 & 11.8 & 0.2 \\
\hline HT3 $(26 \%)^{(1)}$ & 87.4 & 12.2 & 0.4 \\
\hline HT4 $(59 \%)^{(1)}$ & 87.4 & 12.6 & 0.0 \\
\hline Jet $A 1^{(2)}$ & 85.4 & 14.6 & 0.0 \\
\hline
\end{tabular}

${ }^{(1)}$ From GC-MS, ${ }^{(2)}$ From CHN analysis 
Table 7S. Measured properties of pure fuels

\begin{tabular}{|c|c|c|c|c|c|c|c|c|c|c|}
\hline & $\begin{array}{l}\text { Density } \\
\left(\mathrm{kg} / \mathrm{m}^{3}\right)\end{array}$ & $\begin{array}{l}\text { Kinematic } \\
\text { viscosity at } \\
-20^{\circ} \mathrm{C} \\
\left(\mathrm{mm}^{2} / \mathrm{s}\right)\end{array}$ & $\begin{array}{l}\text { Kinematic } \\
\text { viscosity at } \\
40^{\circ} \mathrm{C} \\
\left(\mathrm{mm}^{2} / \mathrm{s}\right)\end{array}$ & $\begin{array}{l}\text { Higher } \\
\text { heating } \\
\text { value } \\
(\mathrm{MJ} / \mathrm{kg})\end{array}$ & $\begin{array}{l}\text { Lower } \\
\text { heating } \\
\text { value } \\
\text { (MJ/kg) }\end{array}$ & $\begin{array}{l}\text { WSD } \\
(\mu m)\end{array}$ & $\begin{array}{l}\text { Flash } \\
\text { point }\left({ }^{\circ} \mathrm{C}\right)\end{array}$ & $\begin{array}{l}\text { Pour point } \\
\left({ }^{\circ} \mathrm{C}\right)\end{array}$ & $\operatorname{COT}\left({ }^{\circ} \mathrm{C}\right)$ & $S P(m m)$ \\
\hline Turpentine & $864.3 \pm 0.9$ & $4.21 \pm 0.01$ & $1.35 \pm 0.00$ & $44.9 \pm 0.0$ & $42.3 \pm 0.0$ & $461 \pm 11$ & $37.3 \pm 1.2$ & $-101.7 \pm 2.9$ & $<-90.0$ & $9.0 \pm 0.0$ \\
\hline$\alpha$-Pinene & $858.4 \pm 1.4$ & $3.79 \pm 0.00$ & $1.29 \pm 0.01$ & $45.4 \pm 0.1$ & $42.8 \pm 0.1$ & $829 \pm 7$ & $34.7 \pm 0.6$ & $-117.0 \pm 0.0$ & $<-90.0$ & $10.0 \pm 0.0$ \\
\hline$\beta$-Pinene & $867.6 \pm 1.1$ & $4.97 \pm 0.01$ & $1.42 \pm 0.01$ & $45.5 \pm 0.0$ & $43.0 \pm 0.0$ & 788 & $37.2 \pm 0.6$ & $-90.0 \pm 0.0$ & $<-90.0$ & $11.7 \pm 0.6$ \\
\hline D-Limonene & $840.2 \pm 0.8$ & $2.16 \pm 0.01$ & $0.86 \pm 0.01$ & $45.1 \pm 0.1$ & $42.6 \pm 0.1$ & $748 \pm 14$ & $52.8 \pm 0.6$ & $-119.0 \pm 0.0$ & $<-90.0$ & $14.7 \pm 0.6$ \\
\hline Myrcene & $793.2 \pm 1.5$ & $2.93 \pm 0.04$ & $1.06 \pm 0.01$ & $46.3 \pm 0.5$ & $43.7 \pm 0.5$ & 537 & $42.2 \pm 0.6$ & n.d. & $<-90.0$ & n.d. \\
\hline HT1 $(3 \%)$ & $863.0 \pm 0.5$ & $4.41 \pm 0.03$ & $1.36 \pm 0.00$ & $44.9 \pm 0.1$ & $42.4 \pm 0.0$ & $364 \pm 19$ & $25.7 \pm 0.6$ & n.d. & $<-90.0$ & $9.3 \pm 0.6$ \\
\hline HT2 (16\%) & $862.2 \pm 0.1$ & $4.58 \pm 0.02$ & $1.42 \pm 0.00$ & $45.0 \pm 0.1$ & $42.5 \pm 0.1$ & $404 \pm 12$ & $24.5 \pm 0.0$ & n.d. & $<-90.0$ & $10.0 \pm 0.0$ \\
\hline HT3 (26\%) & $861.7 \pm 0.5$ & $4.93 \pm 0.03$ & $1.47 \pm 0.00$ & $45.1 \pm 0.1$ & $42.5 \pm 0.1$ & $356 \pm 6$ & $33.2 \pm 0.6$ & n.d. & $<-90.0$ & $10.7 \pm 0.6$ \\
\hline HT4 (59\%) & $859.7 \pm 0.5$ & $5.96 \pm 0.01$ & $1.70 \pm 0.00$ & $45.5 \pm 0.1$ & $42.8 \pm 0.1$ & $505 \pm 1$ & $35.2 \pm 0.3$ & n.d. & $<-90.0$ & $14.7 \pm 0.6$ \\
\hline Jet A1 & $783.6 \pm 0.3$ & $3.09 \pm 0.03$ & $1.09 \pm 0.00$ & $46.3 \pm 0.1$ & $43.2 \pm 0.1$ & 640 & $42.5 \pm 0.0$ & $-64.0 \pm 1.4$ & $-69.2 \pm 0.1$ & $22.3 \pm 0.6$ \\
\hline
\end{tabular}


Table 8S. Estimated properties of pure fuels

\begin{tabular}{|l|l|l|l|l|l|}
\hline & $\begin{array}{l}\text { Mean } \\
\text { molecular } \\
\text { formula }\end{array}$ & $\begin{array}{l}\text { Molecular } \\
\text { weight } \\
\text { (kg/kmol) }\end{array}$ & H/C ratio & $\begin{array}{l}\text { Fuel/air } \\
\text { stoichiometric } \\
\text { ratio }\end{array}$ & TSI \\
\hline Turpentine & $\mathrm{C}_{10.19} \mathrm{H}_{16.30} \mathrm{O}_{0.00}$ & 138.85 & 1.60 & $1 / 14.08$ & $23.2 \pm 0.0$ \\
\hline$\alpha$-Pinene & $\mathrm{C}_{10.00} \mathrm{H}_{16.00} \mathrm{O}_{0.00}$ & 136.23 & 1.60 & $1 / 14.09$ & $20.7 \pm 0.0$ \\
\hline$\beta$-Pinene & $\mathrm{C}_{10.00} \mathrm{H}_{16.00} \mathrm{O}_{0.00}$ & 136.23 & 1.60 & $1 / 14.09$ & $18.0 \pm 0.8$ \\
\hline D-Limonene & $\mathrm{C}_{10.00} \mathrm{H}_{16.00} \mathrm{O}_{0.00}$ & 136.23 & 1.60 & $1 / 14.09$ & $14.6 \pm 0.5$ \\
\hline Myrcene & $\mathrm{C}_{10.00} \mathrm{H}_{16.00} \mathrm{O}_{0.00}$ & 136.23 & 1.60 & $1 / 14.09$ & n.d. \\
\hline HT1 (3\%) & $\mathrm{C}_{10.18} \mathrm{H}_{16.34} \mathrm{O}_{0.00}$ & 138.85 & 1.61 & $1 / 14.09$ & $22.5 \pm 1.3$ \\
\hline HT2 (16\%) & $\mathrm{C}_{10.23} \mathrm{H}_{16.41} \mathrm{O}_{0.01}$ & 139.64 & 1.60 & $1 / 14.07$ & $21.2 \pm 0.0$ \\
\hline HT3 (26\%) & $\mathrm{C}_{10.16} \mathrm{H}_{16.88} \mathrm{O}_{0.03}$ & 139.64 & 1.66 & $1 / 14.11$ & $20.0 \pm 1.0$ \\
\hline HT4 $(59 \%)$ & $\mathrm{C}_{10.06} \mathrm{H}_{17.33} \mathrm{O}_{0.00}$ & 138.30 & 1.72 & $1 / 14.27$ & $14.8 \pm 0.5$ \\
\hline Jet A1 & $\mathrm{C}_{10.76} \mathrm{H}_{21.84} \mathrm{O}_{0.00}$ & $151.24(1)$ & 2.03 & $1 / 14.71$ & $11.1 \pm 0.2$ \\
\hline
\end{tabular}

(1) Calculated following Reference $1 \mathrm{~S}$

Table 9S. Measured properties of blends of turpentine and hydroturpentines with Jet AI

\begin{tabular}{|l|l|l|l|l|}
\hline & $\begin{array}{l}\text { Flash point } \\
\left({ }^{\circ} \boldsymbol{C}\right)\end{array}$ & $\boldsymbol{C O T}\left({ }^{\circ} \boldsymbol{C}\right)$ & $\boldsymbol{S P}(\boldsymbol{m m})$ & TSI \\
\hline T $(2.5 \% \mathrm{v} / \mathrm{v})$ & $41.5 \pm 0.0$ & $-70.1 \pm 0.1$ & $22.3 \pm 0.0$ & $11.2 \pm 0.0$ \\
\hline T $(5 \% \mathrm{v} / \mathrm{v})$ & $41.5 \pm 0.0$ & $-70.5 \pm 0.1$ & $21.3 \pm 0.0$ & $11.6 \pm 0.0$ \\
\hline T $(10 \% \mathrm{v} / \mathrm{v})$ & $41.5 \pm 0.0$ & $-72.0 \pm 0.1$ & $20.9 \pm 0.6$ & $11.7 \pm 0.3$ \\
\hline T $(20 \% \mathrm{v} / \mathrm{v})$ & $40.8 \pm 0.6$ & $-72.7 \pm 0.1$ & $18.9 \pm 0.6$ & $12.7 \pm 0.4$ \\
\hline T $(30 \% \mathrm{v} / \mathrm{v})$ & $39.8 \pm 0.6$ & $-75.2 \pm 0.0$ & $18.2 \pm 0.0$ & $13.0 \pm 0.0$ \\
\hline HT1 $(2.5 \% \mathrm{v} / \mathrm{v})$ & $41.0 \pm 0.0$ & $-69.9 \pm 0.0$ & $23.6 \pm 0.6$ & $10.6 \pm 0.2$ \\
\hline HT1 $(5 \% \mathrm{v} / \mathrm{v})$ & $40.3 \pm 0.6$ & $-70.0 \pm 0.0$ & $23.0 \pm 0.6$ & $10.9 \pm 0.2$ \\
\hline HT1 $(10 \% \mathrm{v} / \mathrm{v})$ & $38.5 \pm 0.0$ & $-71.3 \pm 0.1$ & $22.0 \pm 0.6$ & $11.3 \pm 0.3$ \\
\hline HT1 $(20 \% \mathrm{v} / \mathrm{v})$ & $36.0 \pm 0.0$ & $-72.7 \pm 0.0$ & $20.3 \pm 0.0$ & $12.0 \pm 0.0$ \\
\hline HT1 $(30 \% \mathrm{v} / \mathrm{v})$ & $34.2 \pm 0.6$ & $-75.5 \pm 0.0$ & $17.9 \pm 0.6$ & $13.3 \pm 0.4$ \\
\hline HT2 $(2.5 \% \mathrm{v} / \mathrm{v})$ & $40.5 \pm 0.0$ & $-69.6 \pm 0.1$ & $24.3 \pm 0.0$ & $10.4 \pm 0.0$ \\
\hline HT2 $(5 \% \mathrm{v} / \mathrm{v})$ & $39.5 \pm 0.0$ & $-70.0 \pm 0.0$ & $22.6 \pm 0.6$ & $11.0 \pm 0.2$ \\
\hline HT2 $(10 \% \mathrm{v} / \mathrm{v})$ & $39.0 \pm 0.0$ & $-72.0 \pm 0.0$ & $21.6 \pm 0.6$ & $11.4 \pm 0.3$ \\
\hline HT2 $(20 \% \mathrm{v} / \mathrm{v})$ & $35.5 \pm 0.9$ & $-73.5 \pm 0.0$ & $19.3 \pm 0.0$ & $12.5 \pm 0.0$ \\
\hline HT2 $(30 \% \mathrm{v} / \mathrm{v})$ & $33.7 \pm 0.3$ & $-75.0 \pm 0.0$ & $17.2 \pm 0.0$ & $13.7 \pm 0.0$ \\
\hline HT3 $(2.5 \% \mathrm{v} / \mathrm{v})$ & $41.0 \pm 0.0$ & $-69.4 \pm 0.1$ & $24.3 \pm 0.0$ & $10.4 \pm 0.0$ \\
\hline HT3 $(5 \% \mathrm{v} / \mathrm{v})$ & $41.3 \pm 0.6$ & $-71.2 \pm 0.1$ & $23.6 \pm 0.6$ & $10.6 \pm 0.2$ \\
\hline HT3 $(10 \% \mathrm{v} / \mathrm{v})$ & $41.0 \pm 0.0$ & $-72.0 \pm 0.0$ & $22.6 \pm 0.6$ & $11.0 \pm 0.2$ \\
\hline HT3 $(20 \% \mathrm{v} / \mathrm{v})$ & $39.0 \pm 0.0$ & $-73.3 \pm 0.0$ & $20.3 \pm 0.0$ & $12.0 \pm 0.0$ \\
\hline HT3 $(30 \% \mathrm{v} / \mathrm{v})$ & $38.3 \pm 0.6$ & $-75.5 \pm 0.0$ & $19.3 \pm 0.0$ & $12.4 \pm 0.0$ \\
\hline HT4 $(2.5 \% \mathrm{v} / \mathrm{v})$ & $41.0 \pm 0.0$ & $-69.4 \pm 0.0$ & $24.7 \pm 0.6$ & $10.3 \pm 0.2$ \\
\hline HT4 $(5 \% \mathrm{v} / \mathrm{v})$ & $41.0 \pm 0.0$ & $-69.9 \pm 0.1$ & $24.3 \pm 0.0$ & $10.4 \pm 0.0$ \\
\hline HT4 $(10 \% \mathrm{v} / \mathrm{v})$ & $41.0 \pm 0.0$ & $-72.0 \pm 0.0$ & $23.3 \pm 0.0$ & $10.7 \pm 0.0$ \\
\hline HT4 $(20 \% \mathrm{v} / \mathrm{v})$ & $40.0 \pm 0.0$ & $-73.8 \pm 0.1$ & $22.0 \pm 0.6$ & $11.2 \pm 0.3$ \\
\hline HT4 $(30 \% \mathrm{v} / \mathrm{v})$ & $39.3 \pm 0.6$ & $-75.6 \pm 0.0$ & $20.6 \pm 0.6$ & $11.7 \pm 0.3$ \\
\hline
\end{tabular}


Table 10S. Measured COT of blends of terpenes with Jet A1

\begin{tabular}{|l|l|}
\hline & COT $\left(^{\circ} \boldsymbol{C}\right)$ \\
\hline$\alpha$-Pinene $(2.5 \% \mathrm{v} / \mathrm{v})$ & $-69.6 \pm 0.0$ \\
\hline$\alpha$-Pinene $(5 \% \mathrm{v} / \mathrm{v})$ & $-70.1 \pm 0.0$ \\
\hline$\alpha$-Pinene $(10 \% \mathrm{v} / \mathrm{v})$ & $-71.1 \pm 0.0$ \\
\hline$\alpha$-Pinene $(20 \% \mathrm{v} / \mathrm{v})$ & $-73.2 \pm 0.1$ \\
\hline$\alpha$-Pinene $(30 \% \mathrm{v} / \mathrm{v})$ & $-75.4 \pm 0.0$ \\
\hline$\beta$-Pinene $(2.5 \% \mathrm{v} / \mathrm{v})$ & $-70.0 \pm 0.0$ \\
\hline$\beta$-Pinene $(5 \% \mathrm{v} / \mathrm{v})$ & $-70.2 \pm 0.1$ \\
\hline$\beta$-Pinene $(10 \% \mathrm{v} / \mathrm{v})$ & $-70.8 \pm 0.0$ \\
\hline$\beta$-Pinene $(20 \% \mathrm{v} / \mathrm{v})$ & $-73.0 \pm 0.0$ \\
\hline$\beta$-Pinene $(30 \% \mathrm{v} / \mathrm{v})$ & $-74.4 \pm 0.0$ \\
\hline D-Limonene $(2.5 \% \mathrm{v} / \mathrm{v})$ & $-69.3 \pm 0.1$ \\
\hline D-Limonene $(5 \% \mathrm{v} / \mathrm{v})$ & $-69.8 \pm 0.0$ \\
\hline D-Limonene $(10 \% \mathrm{v} / \mathrm{v})$ & $-71.0 \pm 0.0$ \\
\hline D-Limonene $(20 \% \mathrm{v} / \mathrm{v})$ & $-73.5 \pm 0.1$ \\
\hline D-Limonene $(30 \% \mathrm{v} / \mathrm{v})$ & $-74.4 \pm 0.1$ \\
\hline Myrcene $(2.5 \% \mathrm{v} / \mathrm{v})$ & $-69.3 \pm 0.0$ \\
\hline Myrcene $(5 \% \mathrm{v} / \mathrm{v})$ & $-70.1 \pm 0.0$ \\
\hline Myrcene $(10 \% \mathrm{v} / \mathrm{v})$ & $-70.6 \pm 0.0$ \\
\hline Myrcene $(20 \% \mathrm{v} / \mathrm{v})$ & $-71.6 \pm 0.1$ \\
\hline Myrcene $(30 \% \mathrm{v} / \mathrm{v})$ & $-73.0 \pm 0.1$ \\
\hline
\end{tabular}

Figure 3S. Absolute rate of decrease of viscosity with temperature vs. conversion

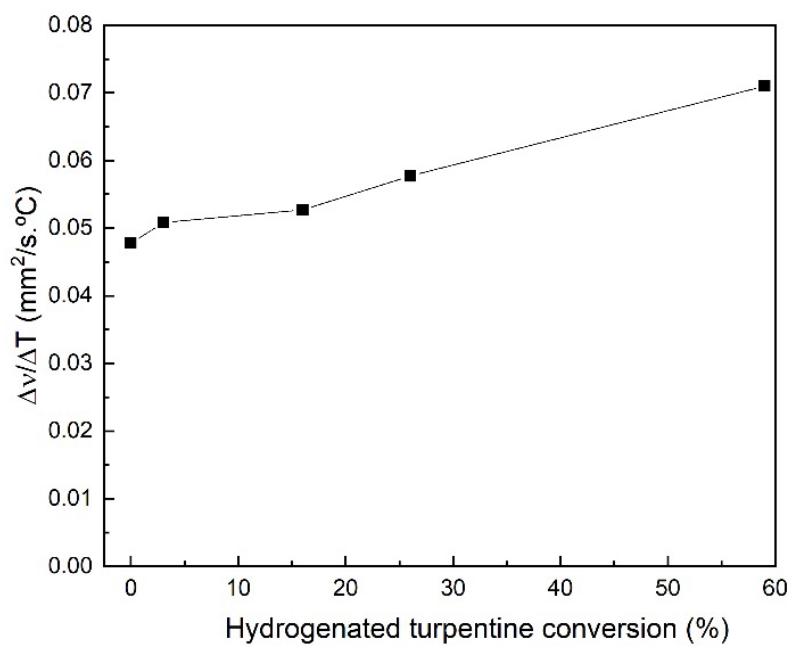


Figure 4S. Distillation curves

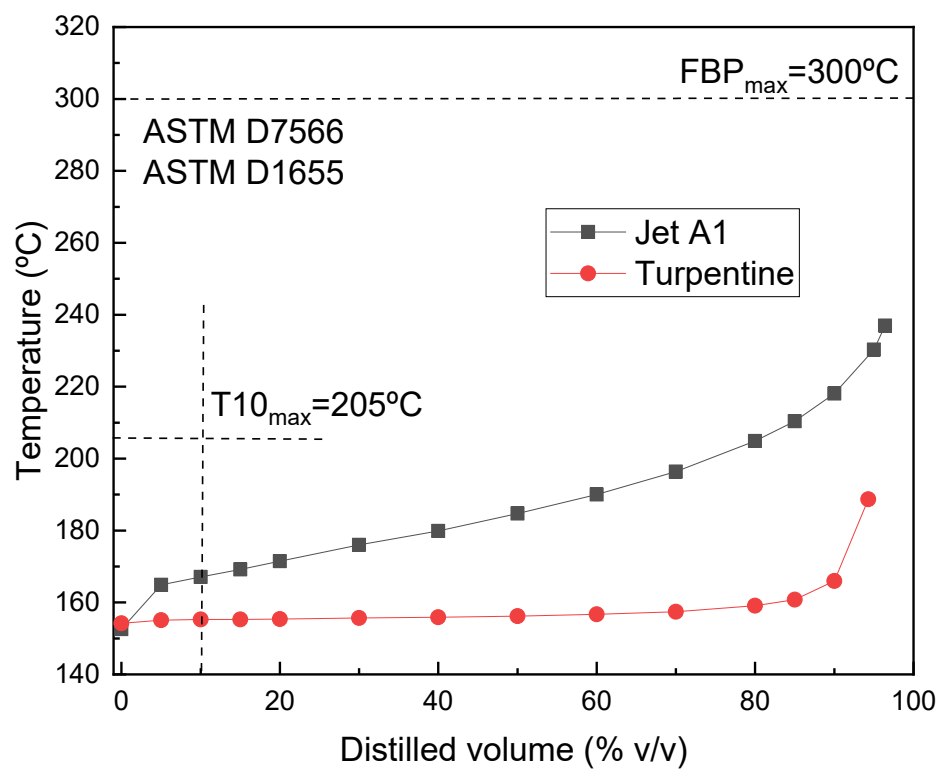

\section{References}

1S. Riazi, M. R., Daubert, T. E. Characterization parameters for petroleum fractions. Industrial and Engineering Chemistry Research, 1987, 26, 755-759. https://doi.org/10.1021/ie00064a023 